\title{
The need for a cross-cultural empirical musicology
}

\author{
MARC LEMAN \\ IPEM, Dept. of Musicology, Ghent University
}

\begin{abstract}
The paper by Lara Pearson shows that a case study based on qualitative description may reveal interesting aspects about the co-occurrence of hand gestures and singing in a particular music culture. However, above all the paper lets us dream about what could be possible if forces from cultural studies and music cognition research were to be combined. A cross-cultural empirical musicology holds the promise of scientific work that goes far beyond qualitative descriptions.
\end{abstract}

Submitted 2013 May 15; accepted 2013 May 27.

KEYWORDS: musical gestures

\section{INTRODUCTION}

LARA Pearson provides a qualitative case study analysis of the use of hand gestures during a fifteenminute vocal lesson given by one single teacher in the Karnatak music tradition. The method is based on a verbal description of how the pitch and gesture trajectories obtained in the study go together. As I understood the paper, the data support the hypothesis that hand gestures co-occur with singing in a consistent way, at least during these fifteen minutes of a single teacher's lesson. However, I am not convinced that the study provides any evidence that warrants a conclusion such as: "The relationship between wide vocal oscillations and extremes of gestural movement found in this study raises the possibility that the gestures observed could be linked to the intensity of muscular movement in the larynx of the vocalist." I believe that the method used does not capture physical effort during singing, and does not provide any evidence about the muscles of the larynx.

Pearson's paper follows a trend in cultural studies to consider music as fully embodied, and that is an interesting viewpoint to start from (Leman, 2008). However, I must confess that Pearson's paper has raised in me a number of doubts and questions about the claimed scientific power of the analysis. I think that it is indeed possible to go beyond the kind of qualitative description that we know from ethnography, but this requires a more advanced use of methods, and perhaps, as I will argue, more effort in collaborative interaction between ethnographers and empirical and systematic musicologists. The issue is that Lara Pearson wants to do more than just carry out a qualitative description. She wants to formulate a hypothesis for which she searches for evidence and, by doing this, she goes beyond the mere ethnography and subscribes to a domain that appeals to cognitive empirical music research. Let me further add that I have the highest appreciation for somebody who takes the risk of crossing the boarders of her own discipline. Therefore, my critique should be read as an attempt to support this kind of work, and to improve it. Admittedly, my viewpoint is highly biased but the main point I want to make is that the paper challenges us to set up closer collaborations in cultural and empirical musicology. The paper by Lara Pearson convinces me that there is an urgent need for this type of collaboration, and that it is in the interests of both the cultural music researcher as well as the systematic music researcher to achieve it.

In what follows I shall argue that finding a good tradeoff between a profound gesture analysis and a profound cultural study is the challenge and the goal of an emerging discipline of cross-cultural empirical musicology. I will clarify my point of view in two brief sections. In the first section I focus on the challenge of musical gesture analysis. In the second section I focus on the call for a cross-cultural empirical musicology. 


\section{THE CHALLENGE OF MUSICAL GESTURE ANALYSIS}

The first question we have to ask ourselves is: What do we really want to show when studying gestures in relation to music? For example, do we want to find support for the hypothesis that gestures co-occur with singing? Lara Pearson seems to suggest that we should show that the use of musical gestures is consistent in the sense that typical musical phrasings co-occur with typical hand gestures. However, what about the different contexts in which this happens (e.g., teaching versus concert playing), and does it count for different teachers, for different regions? Can we find support for such a hypothesis in a single case study? And assuming that we find evidence for this hypothesis on a large scale, what can we do with that knowledge?

Matthew Rahaim in his book on gesture and voice in Hindustani music (Rahaim, 2012) comes to the conclusion that although there is evident gestural mirroring in vocal lessons, the gestures reproduced by students are not necessarily replicas of the teacher's. He suggests that a method for gesturing may be inherited, thus having a social origin that somehow drives the gesture-singing associations of a particular performer. What is the nature of these lineages, which worldview do they subsume, and how do they relate to the audio-motor associations of each performer? Rahaim shows that case studies based on "inside" knowledge can contribute to an understanding of these questions.

I would like to add here that a cross-cultural investigation of gesture and voice could be most interesting. Consider Katty Kochman's study of the use of gestures in vocal pedagogy of opera singing (Kochman, Moelants, \& Leman, in review). Her 'inside' knowledge as an opera singer gives her a privileged access to the study of gestures in Western vocal pedagogy. However, in this study it was not necessary to go deep into the micro-timing details of the gesture-music association as the goal was to identify concepts and metaphors behind supporting gestures. In that sense, the approach is more akin to Rahaim's, where the work is less elaborate at the micro-timing level and more directed towards a qualitative description of the performer's intentions and practices. What this shows is that the type of description should be carefully considered and placed in relation to the goal of the study.

Obviously, there are other studies that go deeper into the micro-timing analysis of musical gestures. For example, Frank Desmet studied gestural segments of a clarinet player in relation to target notes and supporting notes in the music (Desmet, Nijs, Demey, Lesaffre, Martens, \& Leman, 2012). Desmet focused on a methodological framework for gesture analysis that combines bottom-up statistical analysis of gestures (e.g., including an automatic segmentation procedure) with a top-down manual analysis of music. He then tried to find co-occurrences between particular types of gestures (e.g., circular figures) and particular notes or note configurations that function as target notes or support notes. The latter are identified on the basis of 'inside' knowledge that was obtained by involving the performer in the analysis. The focus here is on trying to define a quantitative methodology for gesture analysis. At my lab, this approach is currently further explored by Denis Amelynck, who focuses on the statistical description of the micro-timing of movements and on bottom-up approaches that allow the specification of, for example, goal points in hand gestures that perform a pattern in response to music (Amelynck, Maes, Martens, \& Leman, submitted; see also Naveda and Leman (2010) for spatiotemporal representations that link gesture with music). What we try to gain with this is accuracy of description, theory refinement, explanatory power, perhaps prediction of musical gestural behavior, and modeling. We aim at musical knowledge that has a proactive character and that can for example be used in education or in new applications (see Nijs, Moens, Lesaffre, \& Leman, 2012; Maes, Amelynck, Lesaffre, Arvind, \& Leman, 2013). What I claim is that by studying musical gestures in other cultures, we may broaden and deepen our understanding of musical gestures in these respects, with the goal of obtaining useful information, e.g., for vocal pedagogy. In this area there is an urgent need for 'inside' knowledge about music and gestures.

The challenge that Lara Pearson sets herself is that she wants to relate her 'inside' knowledge to a hypothesis and a descriptive approach, but the hypothesis needs refinement and the descriptive approach does not go far enough in my opinion. There is an interesting passage where she says, "Gesture here seems to act as a schema of the phrase as a whole, and could perhaps be viewed as a kinetic control system for the complex and detailed array of patterns encountered in the music [...].". But this is a distant goal for her. She immediately adds that: "To build on this observation, further studies should be conducted in order to gather more data on gesture/musical-feature correspondence and variation over several lessons given by a single performer in both śankarābharanam and other rāgas." Yet I believe that this type of hypothesis may be more interesting than describing co-occurrences. The reason is that such a hypothesis has a tight connection with strong explanatory and proactive theories. In fact, it reminds me of Andy Clark (2008) who 
suggested that gestures can be understood as embodied extensions of our mind and that these embodied extensions can be of benefit to an ongoing cognitive process. Also Rahaim remarks that by gestural enacting, teachers present a kinetic model for how the 'musicking' should proceed (Rahaim, 2012, p. 115). This is interesting material for cognitive musicology, and it ties up with theories of embodied cognition, action-perception and sensorimotor coupling. Applied to Lara Pearson's case study one could ask whether the gestures that accompany the singing performance may be seen as embodied extensions of a mental concept that allows the active structuring (in terms of sensory visual and proprioceptive motor input) that guides the ongoing activity of another motor activity (namely the singing itself). In addition, by bringing gestures into a physical space, the extended mind of the performer can be socially shared in a multi-modal way (both through gesture and singing) and thus be accessible to others who may engage with it in an empathic relationship (e.g., through gestural imitation). Understanding this mechanism would be highly valuable for vocal pedagogy and 'inside' knowledge from different cultures would be highly important to deepen our understanding. It would contribute to a better understanding of a cognitive dynamics at work in different cultural environments.

In short, we should try to link 'inside' knowledge with challenging (proactive and perhaps testable) hypotheses. And I do not know whether we need more data, but we definitely need good data and, above all, a good specification of what we really want to investigate (Schneider, 1993, 2006; Honing, 2004, 2006; Parncutt 2007; Leman, 2010a, 2010b). Going beyond qualitative descriptions for musical gesture means that the problem definition should be clarified and that some hypotheses should be formulated that connects to appealing theories, or in which advanced modeling techniques can be used for the analysis and synthesis of gestures (Godøy \& Leman, 2010; Lesaffre and Leman, 2013).

\section{TOWARDS A CROSS-CULTURAL EMPIRICAL MUSICOLOGY}

Pearson's attempt suggests that fieldwork may lead to interesting viewpoints that clarify the different possible ways, values and intentions that influence human interaction with music. This kind of 'inside' knowledge is needed in order to be able to understand the observed musical phenomena in a scientific perspective. Her willingness to adopt tools and methods from empirical or systematic musicology in order to enrich the qualitative descriptions is appealing. Her study should therefore be read as an attempt to expand ethnography in this direction. However, it is not something that one person seems to be able to manage on her own. I am very much in favor of the idea that we should develop a discipline of crosscultural empirical musicology in which the best of the two worlds can be combined.

\section{CONCLUSIONS}

The paper by Lara Pearson provides a starting point to think about the common interest of cultural studies and music cognition research to join forces and to go beyond the qualitative case study analysis approach. With my critique, inspired by Pearson's attempt, I hope to contribute to this discussion.

\section{REFERENCES}

Amelynck, D., Maes, P., Martens, J.-P., \& Leman, M. (submitted). Low dimensional representation of expressiveness in music-driven spontaneous dance movements.

Clark, A. (2008). Supersizing the Mind: Embodiment, Action, and Cognitive Extension. Oxford: Oxford University Press.

Desmet, F., Nijs, L., Demey, M., Lesaffre, M., Martens, J.-P., \& Leman, M. (2012). Assessing a clarinet player's performer gestures in relation to locally intended musical targets. Journal of New Music Research, Vol. 41, No. 1, pp. 31-48.

Godøy, R.I., \& Leman, M. (Eds.) (2010). Musical Gestures: Sound, Movement, and Meaning. New York: Routledge. 
Honing, H. (2004). The comeback of systematic musicology: New empiricism and the cognitive revolution. Tijdschrift voor Muziektheorie, Vol. 9, No. 3, pp. 241-244.

Honing, H. (2006). On the growing role of observation, formalization and experimental method in musicology. Empirical Musicology Review, Vol. 1, No. 1, pp. 2-6.

Kochman, K., Moelants, D., \& Leman, M. (in review). The role of gestural concatenates as a communicative tool in vocal pedagogy. Journal of Interdisciplinary Music Studies.

Leman, M. (2008). Embodied Music Cognition and Mediation Technology. Cambridge, MA: The MIT Press.

Leman, M. (2010a). Some reflections on systematic musicology as proactive science. In: R. Bader, C. Neuhaus, \& U. Morgenstern (Eds.), Concepts, Experiments, and Fieldwork: Studies in Systematic Musicology and Ethnomusicology. Frankfurt am Main: Peter Lang, pp. 21-34.

Leman, M. (2010b). Systematic musicology at the crossroads of modern music research. In: A. Schneider (Ed.), Systematic and Comparative Musicology: Concepts, Methods, Findings. Hamburger Jahrbuch für Musikwissenschaft, Vol. 24. Frankfurt am Main: Peter Lang, pp. 89-115.

Lesaffre, M., \& Leman, M. (Eds.) (2013). The Power of Music: Researching Musical Experiences. Leuven: ACCO.

Maes, P.J., Amelynck, D., Lesaffre, M., Arvind, D.K., \& Leman, M. (2013). The "Conducting Master": an interactive, real-time gesture monitoring system based on spatiotemporal motion templates. International Journal of Human-Computer Interaction, Vol. 29, No. 7, pp. 471-487.

Naveda, L., \& Leman, M. (2010). The spatiotemporal representation of dance and music gestures using topological gesture analysis (TGA). Music Perception, Vol. 28, No. 1, pp. 93-111.

Nijs, L., Moens, B., Lesaffre, M., \& Leman, M. (2012). The Music Paint Machine: Stimulating selfmonitoring through the generation of creative visual output using a technology-enhanced learning tool. Journal of New Music Research, Vol. 41, No. 1, pp. 79-101.

Parncutt, R. (2007). Systematic musicology and the history and future of Western musical scholarship. Journal of Interdisciplinary Music Studies, Vol. 1, No. 1, pp. 1-32.

Rahaim, M. (2012). Musicking Bodies: Gesture and Voice in Hindustani Music. Middletown, Connecticut: Wesleyan.

Schneider, A. (1993). Systematische Musikwissenschaft: Traditionen, Ansätze, Aufgaben. Systematische Musikwissenschaft, Vol. 1, No. 1, pp. 145-180.

Schneider, A. (2006). Comparative and systematic musicology in relation to ethnomusicology: A historical and methodological survey. Ethnomusicology, Vol. 50, No. 2, pp. 236-258. 